\title{
A simplify fuzzy logic controller design based safe experimentation dynamics for Pantograph-Cateary system
}

\author{
Mohd Faisal Farhan, Nor Sakinah Abdul Shukor, Mohd Ashraf Ahmad, Mohd Helmi Suid, \\ Mohd Riduwan Ghazali, Mohd Falfazli Mat Jusof \\ Faculty of Electrical and Electronics Engineering, Universitas Malaysia Pahang, Malaysia
}

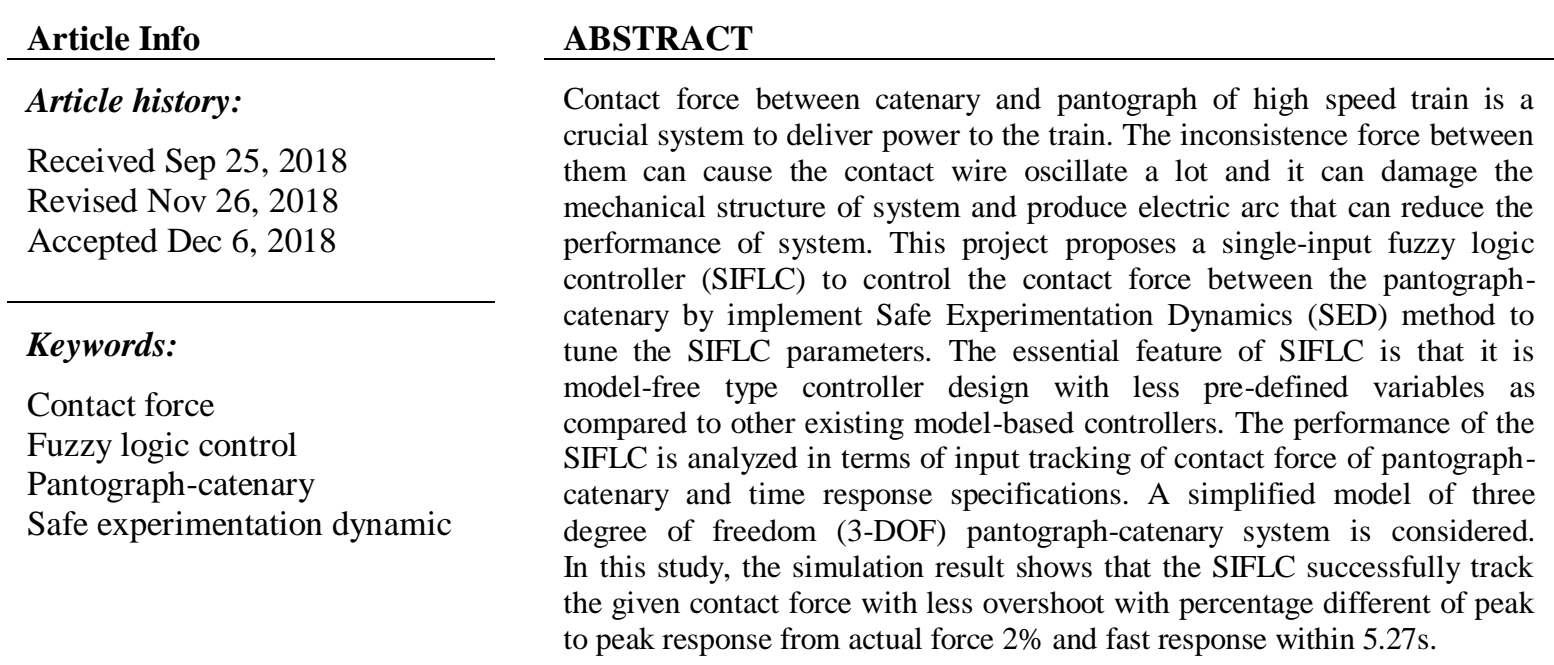

Copyright $\odot 2019$ Institute of Advanced Engineering and Science. All rights reserved.

\section{Corresponding Author:}

Mohd Faisal Farhan bin Kamerul Bieza,

Faculty of Electrical and Electronics Engineering,

Universiti Malaysia Pahang,

26600 Pekan, Pahang, Malaysia.

Email: mohdfaisalfarhan@ump.edu.my

\section{INTRODUCTION}

Public transportation is one of the crucial systems for the community to ease their life day by day. Along the advancement of technologies, some electronic device is use to control the system of the public transport such as high speed train. This electronic device used to optimum the train performance and services. In high speed train system, the functions of electronic control device are to control the contact force between catenary and pantograph. Pantograph-catenary (PAC) system will determine the amount of electricity required to power the motors of an electric train.

The main problem on high-speed train pantograph-catenary system is to control the contact force between them. When there is a sliding movement between the pantograph and catenary, a periodic vibration of the pantograph will form an inconsistence in the contact force between the PAC systems due to the change of stiffness. This inconsistence force will generate an electric arc that can damage the mechanical structure of the PAC systems and reduce the system performance.

In most of the cases, the desired constant contact force that is required by the PAC system in all operating condition is around $100 \mathrm{~N}$ [1]. However, in order to get the actual value for the constant contact force, the counteraction of the variation contact force could be obtained by using several methods. One of the methods is by tightening the contact wire tension to increase the stiffness. Besides, the other method is by increasing modulus of the contact force [2]. The first method actually will be more costly because the railway 
systems need to be connected at the same standard. By considering this situation, other method was implemented which by using a controller to obtain the constant contact force.

So far, there are many controllers have been reported to control the contact force between the catenary and pantograph. There are active control [3], adaptive fuzzy logic control [4] and fuzzy sliding mode controller [5]. Most of the developed controllers are model-based controller that requires an accurate model of the system, while there is always a gap between the model and the real system, such as unmodeled dynamics problem. Moreover, the developed conventional fuzzy logic in [4] requires a lot of effort to find the optimal variables, such as types of membership functions (MFs), range of universal boundary, number of MF and many more. On the other hand, the SIFLC is a good potential on solving above mentioned issues. The SIFLC does not require any knowledge of the plant in its design procedure and it requires less predefined variables than the conventional FLC. In particular, it does not require any rules and membership function. So far, the SIFLC has been implemented in various type of control problems, such as-flexible joint manipulator of robot [6], double pendulum type overhead crane [7], underwater vehicle manipulator system [8] and magnetic levitation system [9].

In this paper, a single input fuzzy logic controller (SIFLC) of pantograph-catenary for high-speed train is proposed. The simplification of conventional FLC is called as a single input fuzzy logic (SIFL). The simplification of SIFLC is formed by using a signed distance approach [10]. The reduction in the number of rules in rule table to one-dimension allowing it to be treated as single input single output (SISO) controller. The tuned parameter for SILFC is determined by using SED method [11]. The proposed SIFLC is tested to a 3-DOF PAC system. The performance of SIFLC is analyzed in terms of trajectory tracking of contact force and its time response specifications.

\section{MODELLING PAC SYSTEM}

The pantograph-catenary system model considered in this work is shown in Figure 1. The mass at the top represent by $m_{1}$, the mass in the middle represent by $m_{2}$ and the lower mass represent by $m_{3}$. In the normal condition, the catenary will be in contact with the pantograph. In this condition $x_{1} \equiv x_{c}$ and the weight of upper block will be $m_{3}+m_{c}$ where the weight of catenary is $m_{c}$. Therefore, the state-space equations are shown in (1) and (2).

$$
\begin{aligned}
& \dot{x}=A x+B u+D w(t) \\
& y=C x,
\end{aligned}
$$

with the vector $x=\left[\begin{array}{llllll}x_{1} & \dot{x}_{1} & x_{2} & \dot{x}_{2} & x_{3} & \dot{x}_{3}\end{array}\right]^{T}$, and matrices $A, B, C$ and $D$ are expressed by,

$$
A(t)=\left[\begin{array}{cccccc}
0 & 1 & 0 & 0 & 0 & 0 \\
-\sigma_{21} & -\sigma_{22} & \frac{k_{2}}{m_{1}} & \frac{b_{2}}{m_{1}} & 0 & 0 \\
0 & 0 & 1 & 1 & 0 & 0 \\
\frac{k_{1}}{m_{2}} & \frac{b_{1}}{m_{2}} & -\sigma_{43} & -\sigma_{44} & \frac{b_{1}}{m_{2}} & \frac{b_{1}}{m_{2}} \\
0 & 0 & 0 & 0 & 0 & 1 \\
0 & 0 & \frac{k_{2}}{m_{3}} & \frac{b_{2}}{m_{3}} & -\sigma_{65} & -\sigma_{66}
\end{array}\right]
$$

where:

$$
\begin{aligned}
& -\sigma_{21}=\frac{k_{1}+k_{c}}{m_{1}+m_{c}}, \\
& -\sigma_{22}=\frac{b_{1}+b_{c}}{m_{1}+m_{c}}, \\
& -\sigma_{43}=\frac{k_{1}+k_{2}}{m_{2}}, \\
& -\sigma_{44}=\frac{b_{1}+b_{2}}{m_{2}}, \\
& -\sigma_{65}=\frac{k_{3}+k_{2}}{m_{3}}, \\
& -\sigma_{66}=\frac{b_{3}+b_{2}}{m_{3}},
\end{aligned}
$$




$$
\begin{aligned}
B & =\left[\begin{array}{llllll}
0 & 0 & 0 & 0 & 0 & \frac{1}{m_{3}}
\end{array}\right]^{T} \\
C & =\left[\begin{array}{llllll}
Q_{1} & Q_{2} & Q_{3} & Q_{4} & 0 & 0
\end{array}\right]^{T} \\
D & =\left[\begin{array}{llllll}
0 & 0 & 0 & 0 & 0 & \frac{1}{m_{3}}
\end{array}\right]^{T}
\end{aligned}
$$

where,

$$
\begin{aligned}
& Q_{1}=\frac{m_{1} \times k_{c}+\left(2 \times m_{1}+m_{c}\right) \times k_{1}}{m_{1}+m_{c}} \\
& Q_{2}=\frac{m_{1} \times b_{c}+\left(2 \times m_{1}+m_{c}\right) \times b_{1}}{m_{1}+m_{c}} \\
& Q_{3}=Q_{4}=\frac{\left(2 \times m_{1}+m_{c}\right) \times k_{1}}{m_{1}+m_{c}}
\end{aligned}
$$

To get more accurate result on modeling the PAC system, mass and damper variation are considered. The catenary is modeled by the system spring, damper and mass. The mass $m_{c}$, spring stiffness $k_{c}$ and damping constant $b_{c}$, exhibit periodic characteristic for each section. Therefore, a Fourier series expansion is considered as shown in (7).

$$
\begin{aligned}
& m_{c}(t)=m_{c 0}+\sum_{i=1}^{3} m_{c i} \cos \left(\frac{2 \pi i}{L} x(t)\right) \\
& b_{c}(t)=b_{c 0}+\sum_{i=1}^{3} b_{c i} \cos \left(\frac{2 \pi i}{L} x(t)\right) \\
& k_{c}(t)=k_{c 0}+\sum_{i=1}^{3} k_{c i} \cos \left(\frac{2 \pi i}{L} x(t)\right)
\end{aligned}
$$

where the tower and the train is separated with actual distance of $x=x(t)$, the span length represent by, $L$. The train speed is represented, $x(t)=V \times t$ where the velocity of train is, $V$ and $t$ is time to reach the distance. For each of the parameter in PAC system, we just estimate it by referring to the previous research paper in [5]. The baseline catenary parameters that have been used in the pantograph and catenary model is shown in Table 1.

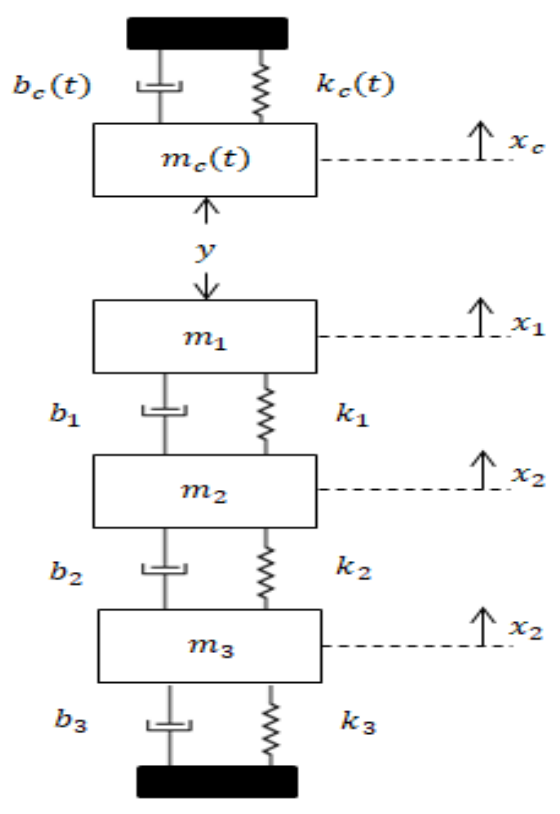

Table 1. Baseline Catenary Parameters [11]

\begin{tabular}{ccc}
\hline Parameter & Notation & Value \\
\hline & $m_{c 1}$ & $195 \mathrm{~kg}$ \\
& $m_{c 1}$ & $100 \mathrm{~kg}$ \\
Catenary Mass & $m_{c 2}$ & $20 \mathrm{~kg}$ \\
& $m_{c 3}$ & $5 \mathrm{~kg}$ \\
& $m_{1}$ & $8.5 \mathrm{~kg}$ \\
& $m_{2}$ & $4.63 \mathrm{~kg}$ \\
& $m_{3}$ & $4.8 \mathrm{~kg}^{-1} \mathrm{~s}$ \\
\hline \multirow{5}{*}{ Catenary Dampers } & $b_{c 0}$ & $24 \mathrm{Nm}^{-1} \mathrm{~s}$ \\
& $b_{c 1}$ & $240 \mathrm{Nm}^{-1} \mathrm{~s}$ \\
& $b_{c 2}$ & $50 \mathrm{Nm}^{-1} \mathrm{~s}$ \\
& $b_{c 3}$ & $12 \mathrm{Nm}^{-1} \mathrm{~S}$ \\
& $b_{1}$ & $5 \mathrm{Nm}^{-1}$ \\
& $b_{2}$ & $5400 \mathrm{Nm}^{-1} \mathrm{~s}$ \\
& $b_{3}$ & $32 \mathrm{Nm}^{-1} \mathrm{~s}$ \\
\hline & $k_{c 0}$ & $7000 \mathrm{Nm}^{-1}$ \\
& $k_{c 1}$ & $3360 \mathrm{Nm}^{-1}$ \\
& $k_{c 2}$ & $650 \mathrm{Nm}^{-1}$ \\
& $k_{c 3}$ & $160 \mathrm{Nm}^{-1}$ \\
& $k_{1}$ & $6045 \mathrm{Nm}^{-1}$ \\
& $k_{2}$ & $50 \mathrm{Nm}^{-1}$ \\
& $k_{3}$ & $1 \mathrm{Nm}^{-1}$ \\
\hline Span Length & $L$ & $65.52 \mathrm{~m}$ \\
\hline
\end{tabular}

Figure 1. PAC system linear model 


\section{SINGLE INPUT FUZZY LOGIC CONTROLLER (SIFLC)}

This section presents the design of SIFLC. Most of the FLC consist of two variable inputs, which are error $(e)$ and error different $(\dot{e})$. Instead of using two input variables $(e, \dot{e})$ to produce the output $\mu_{0}$, it is possible to use a single input variable only. The application of SIFLC will reduce the number of rules compared to the case of conventional Fuzzy Logic controller:

The single input fuzzy logic can be obtained by using a method called signed distance approach. This approach will remove the number of input into single input variable called signed distance. The distance to an actual diagonal line is called signed distance where the input variable $(e, \dot{e})$, come out from diagonal line, $L_{z}$. In other words, to derive the distance variable, $\mathrm{Q}(e, \dot{e})$ need to be an intersection point at $L_{z}$ and perpendicular to operating point $\mathrm{P}(e, \dot{e})[6]$.

From the Figure 2, the straight line function can be represented by $L_{z}$. The equation of the straight line is:

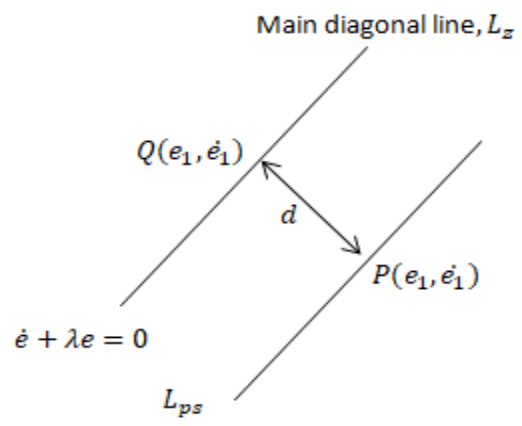

Figure 2. Signed distance approach

$$
\dot{e}+\lambda e=0
$$

From (8), $\lambda$ is the inclination magnitude of the diagonal line $L_{z}$. The distance, $d$ from $\mathrm{P}(e, \dot{e})$ to $\mathrm{Q}(e, \dot{e})$ can be defined as [6].

$$
d=\frac{\dot{e}+\lambda e}{\sqrt{1+\lambda^{2}}}
$$

The result from the derivation of input distance variable will form a one-dimension rule table instead of two-dimension rule table that was used in conventional FLC [10]. The rule table before the input variable derivation process is shown in Table 2 while the simplified rule table is depicted in Table 3 where $L_{L N}, L_{M N}$, $L_{S N}, Z E, L_{S P}, L_{M P}$ and $L_{L P}$ are the diagonal. The diagonal line related to the new input for rule table, while $L N, M N, S N, Z E, S P, M P$ and $L P$ represent the output related to diagonal line. The value of $d$ determines the control action of FLC. Therefore, it is appropriate to call this controller as SIFLC.

Table 2. Two-dimension Rules Table

\begin{tabular}{cccccccc}
\hline $\boldsymbol{e} / \dot{\boldsymbol{e}}$ & LP & MP & SP & ZE & SN & MN & LN \\
\hline LN & ZE & SN & MN & LN & LN & LN & LN \\
MN & SP & ZE & SN & MN & LN & LN & LN \\
SN & MP & SP & ZE & SN & MN & LN & LN \\
ZE & LP & MP & SP & ZE & SN & MN & LN \\
SP & LP & LP & MP & SP & ZE & SN & MN \\
MP & LP & LP & LP & MP & SP & ZE & SN \\
LP & LP & LP & LP & LP & MP & SP & ZE \\
\hline
\end{tabular}

Table 3. One-dimension Rules Table

\begin{tabular}{cccccccc}
\hline $\boldsymbol{d}$ & $\mathrm{L}_{\mathrm{LN}}$ & $\mathrm{L}_{\mathrm{MN}}$ & $\mathrm{L}_{\mathrm{SN}}$ & $\mathrm{L}_{\mathrm{ZE}}$ & $\mathrm{L}_{\mathrm{LP}}$ & $\mathrm{L}_{\mathrm{MP}}$ & $\mathrm{L}_{\mathrm{LP}}$ \\
\hline $\boldsymbol{\mu 0}$ & $\mathrm{LN}$ & $\mathrm{MN}$ & $\mathrm{SN}$ & $\mathrm{ZE}$ & $\mathrm{SP}$ & $\mathrm{MP}$ & $\mathrm{LP}$ \\
\hline
\end{tabular}

Figure 3 shows the structure of control system for pantograph-catenary system in high speed train using SIFLC. Figure 4 shows the detailed structure of SIFLC. It consists of several blocks that is derived from (9). The control surface is designed based on a saturation function that can be amplified by using a gain $r$.

Indonesian J Elec Eng \& Comp Sci, Vol. 14, No. 2, May 2019 : 903 - 911 


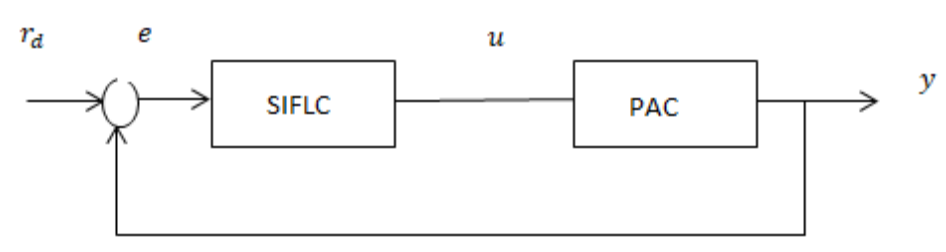

Figure 3. Structure of control system

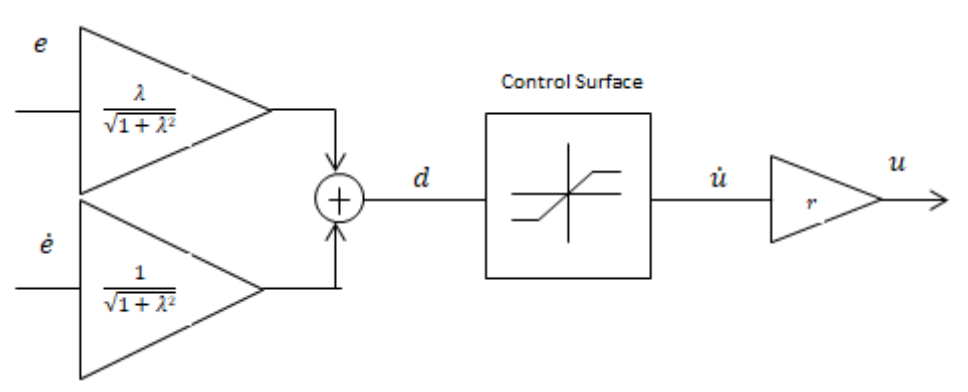

Figure 4. SIFLC structure

\section{SIFLC DESIGN BASED SAFE EXPEIMENTATION DYNAMICS}

A data-driven SIFLC tuning based on SED algorithm is stated in this section.

A. Safe Experimentation Dynamics (Sed)

The problem of the optimization is stated as

$$
\min _{z \in X^{n}} g(z)
$$

where the function represents by $g: X^{n} \rightarrow X$ and $\boldsymbol{z} \in X^{n}$ is parameter design. From (10), the optimal solution $\mathbf{z}^{*} \in X^{n}$ is achieved by updating the parameter design using SED algorithm. The rules in updating SED algorithm is stated as

$$
z_{i}(b+1)=h\left(\overline{z_{l}}-K_{g} r_{2}\right)
$$

where $b=0,1, \ldots$, is iteration number, $z_{i} \in X$ is the $i$ th element of $\boldsymbol{z} \in X^{n}, \bar{z}_{l} \in X$ is the $i$ th iteration of $\bar{z}_{\iota} \in X^{n} \cdot \overline{\boldsymbol{z}}$ used to save current best value of design parameter, $K_{g}$ is the size of interval to determine the random steps on $z_{i} \in X$ and the random number value is $r_{2} \in X$. In (11), the $h$ function is given by

$$
h(.)=\left\{\begin{array}{c}
Z_{\text {max }}, \quad \overline{Z_{l}}-K_{g} r_{2}>Z_{\text {max }} \\
\bar{Z}_{\iota}-K_{g} r_{2}, \quad Z_{\text {min }} \leq \bar{Z}_{l}-K_{g} r_{2} \leq Z_{\text {max }} \\
Z_{\text {min }}, \quad \bar{Z}_{\iota}-K_{g} r_{2}<Z_{\text {min }}
\end{array}\right.
$$

where the pre-determined minimum and maximum parameter design is and $Z_{\min }$ and $Z_{\max }$. The steps procedures to implement SED algorithm are as follows:

Step 1: Define the value of $Z_{\text {min }}, Z_{\text {max }}, K_{g}$ and $E . b$ is set $b=0$ and $\boldsymbol{z}(0)$ set as the initial condition for parameter design. The loss function be $g(\boldsymbol{z}(0))$ and as normal $\overline{\boldsymbol{z}}=\boldsymbol{z}(0)$ and $\bar{g}=g(\boldsymbol{z}(0))$. For a new random setting for $\boldsymbol{z}, E$ is probability that used where it is scalar. To save the loss function present best value, variable $\bar{g}$ is used.

Step 2: When the value $g(\boldsymbol{z}(b))<\bar{g}$, execute $\overline{\mathbf{z}}=\boldsymbol{z}(b)$ and $\bar{g}=g(\boldsymbol{z}(b))$. Apart of that, proceed to next step.

Step 3: Produce random number $r_{1}$. Produce second random number $r_{2}$ if $r_{1}<E$ and by updating the (11), obtained the value of $z_{i}(b+1)$. Otherwise, $z_{i}(b+1)=\bar{z}_{l}$.

Step 4: Achieve the loss function $g\left(z_{i}(b+1)\right)$.

Step 5: If the pre-stated termination condition that based on the designated maximum number of iteration, $b_{\text {max }}$ is satisfied, the algorithm stops with the solution $z^{*}:=\arg \min _{z \in\{z(0), z(1), \ldots, z(b+1)\}} g(z)$. Otherwise, repeat step 2 . 
B. Siflc-Tuned Sed

For this section, the SIFLC-tuned SED design parameter is presented by

$$
\psi=[\lambda r]^{T}
$$

In particular, we set $\psi_{i}=10^{z_{i}}(i=1,2)$ and the loss function is $J(\lambda, r)=\int_{0}^{30} e(t) d t$. Then the SIFLC-tuned SED can be implemented as follows:

Step 1: Consider $g(z)=J(\lambda, r)$ and $z_{i}=\log \psi_{i}$. Then, the maximum iteration, $b_{\max }$ is determine.

Step 2: Execute the SED algorithm in Section 4.1.

Step 3: When reaching $b_{\max }$, attain the optimal output $z^{*}=\bar{z}\left(b_{\max }\right)$. Then implement $\psi^{*}=\left[\begin{array}{ll}10^{z_{1}} & 10^{z_{2}}\end{array}\right]^{T}$ in the SIFLC system in Figure 4.

\section{SIMULATION RESULTS}

In this section, the simulation result of the SIFLC for controlling the contact force between the catenary and pantograph is presented. The SED algorithm parameters are set as $Z_{\max }=1, Z_{\min }=-3$, $E=0.62, K_{g}=0.015$ and $b_{\max }=150$. The train velocity is set as $V=350 \mathrm{~km} / \mathrm{h}$ and the desired input function is given by

$$
r_{d}=50 \tanh (t-2.5)+50
$$

The loss function response when 150 iterations have been used for SED is shown in Figure 5. The loss function is successfully minimized after 150 iterations. Table 4 shows the optimal design parameters. From the results, it exposed that SED method can minimize the loss function.

Table 4. Design Parameter

\begin{tabular}{ccccc}
\hline \multirow{2}{*}{ SIFLC Gain } & \multirow{2}{*}{$z(0)$} & $\begin{array}{c}\psi \text { correspond to } \\
z(0)\end{array}$ & $z(150)$ & $\psi$ correspond to \\
& & & $z(150)$ \\
\hline$\lambda$ & -2 & 0.01 & -1.5318 & 0.0294 \\
$\mathrm{r}$ & 0 & 1 & 0.6330 & 4.2958 \\
\hline
\end{tabular}

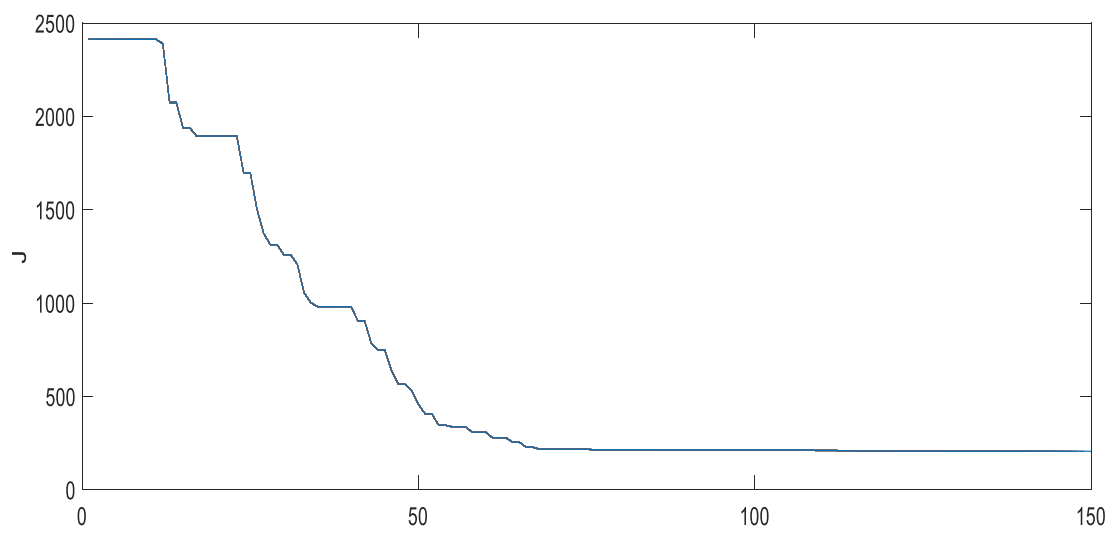

Figure 5. Response of loss function using SED

Next, the response of SIFLC is shown in Figure 6. The blue line represents desired response for the PAC system while the red lines indicate the response after 150 iteration of SED. It shows that the response can track the desired reference signal. There are some oscillations after 3.6 seconds and this result is comparable with the result in [5]. 


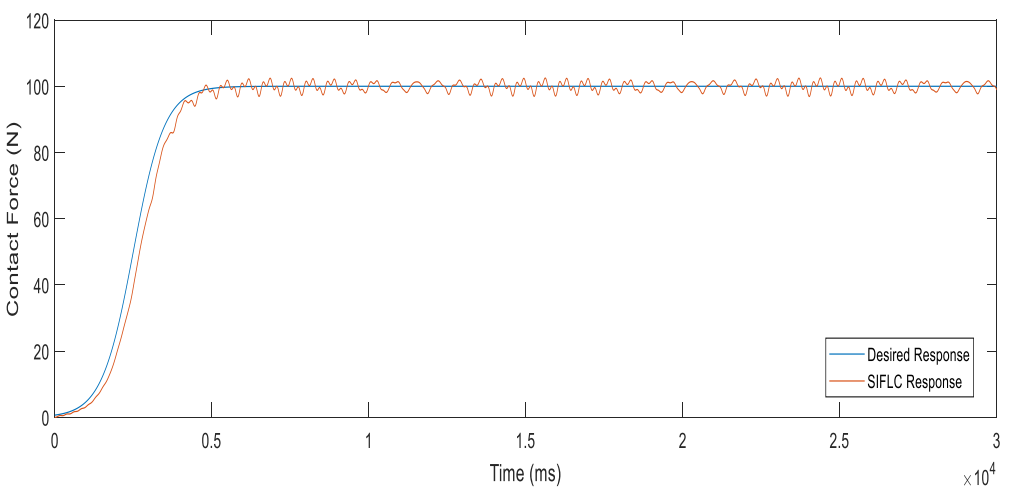

Figure 6. SIFLC performance when tuned by SED

In Figure 7, progress of $x$ denote that permanents contact of the PAC. The observation oscillation shows the vertical movement of pantograph floors. This oscillations influence by parameters that depends on the displacement of the train. Therefore, they simulate the fluctuations that can be determined in actual simulation of PAC.

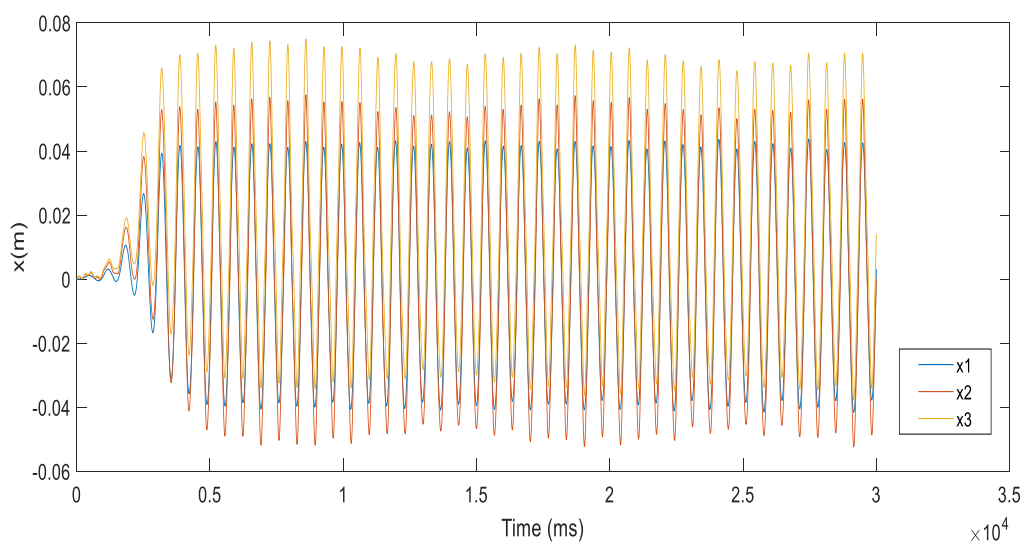

Figure 7. Displacement of the masses of the model

From Figure 8, the performance of SIFLC is compared to PID controller. For SIFLC the response shows it start to response smoothly until reach the desired contact force which is $100 \mathrm{~N}$. On the other hand, the oscillation of the tracking contact force is almost same as compared to SIFLC. As resulted, SIFLC have the fast response as compared to PID controller.

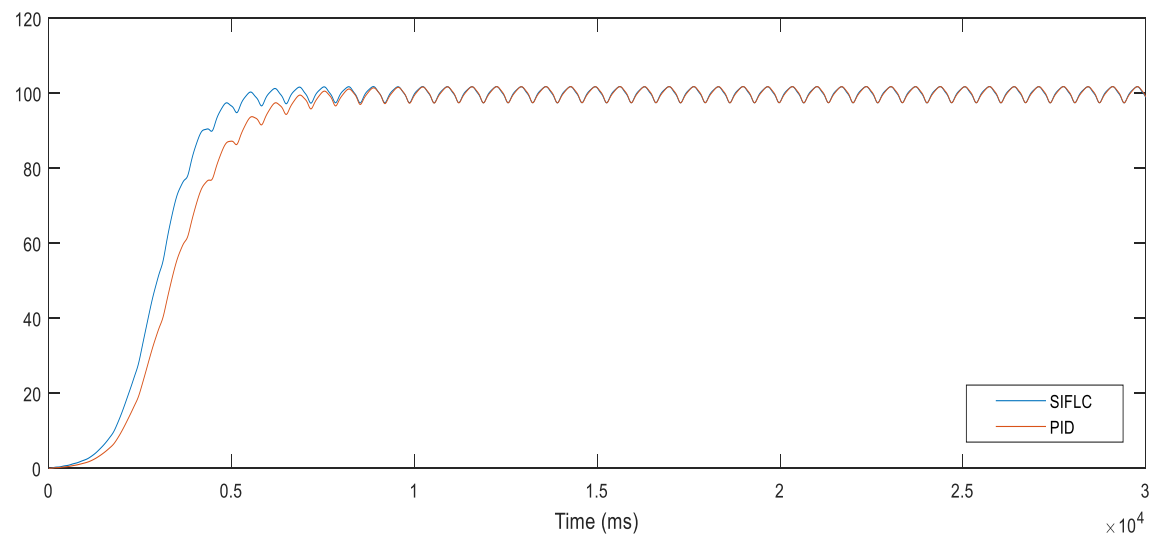

Figure 8. Output tracking for SIFLC and PID controller

A simplify fuzzy logic controller design based safe experimentation dynamics for... (Mohd Faisal Farhan) 
Table 5 shows the controller performance for SIFLC and PID. As shown in the table, SIFLC have a fast response as compared to PID controller because of the rise time to track the desired contact force. However, both controllers have the same percentage overshoot due to the same oscillation on tracking desired contact force.

Table 5. Controller Performance

\begin{tabular}{lcc}
\hline Parameter & SIFLC & PID \\
\hline Rise Time & $5.27 \mathrm{~s}$ & $6.22 \mathrm{~s}$ \\
Percentage Overshoot & $1 \%$ & $1 \%$ \\
Peak to Peak Value of Force at Steady State & $98 \mathrm{~N}$ to $102 \mathrm{~N}$ & $95 \mathrm{~N}$ to $102 \mathrm{~N}$ \\
Percentage Different Between Peak to Peak & $2 \%$ & $3 \%$ \\
\hline
\end{tabular}

\section{CONCLUSION}

From this study, the problem on the inconsistence contact force between the catenary and pantograph is exposed and a solution based on SIFLC is presented and compared with the PID controller performance. The SED algorithm can improve the tuned parameters that need to be used by SIFLC. The simulation is applied to the time variable, consideration of the uncertain pantograph-catenary and a speed of $350 \mathrm{~km} / \mathrm{h}$.

\section{ACKNOWLEDGEMENTS}

This project has been fully supported by UMP research grant PGRS1703104.

\section{REFERENCES}

[1] A. Levant, A. Pisano, and E. Usai, "Output-feedback control of the contact-force in high-speed-train pantographs," in Proceedings of the 40th IEEE Conference on Decision and Control (Cat. No.01CH37228), 2001, vol. 2, pp. 1831-1836 vol.2.

[2] G. Poetsch et al., "Pantograph/catenary dynamics and control," Veh. Syst. Dyn., vol. 28, no. 2-3, pp. 159-195, 1997.

[3] W. Shudong, G. Jingbo, and G. Guosheng, "Research of the active control for high-speed train pantograph," in 2008 IEEE Conference on Cybernetics and Intelligent Systems, 2008, pp. 749-753.

[4] E. Karaköse and M. T. Gençoğlu, "Adaptive fuzzy control approach for dynamic pantograph-catenary interaction," in Proceedings of 15th International Conference MECHATRONIKA, 2012, pp. 1-5.

[5] N. Mokrani and A. Rachid, "A robust control of contact force of pantograph-catenary for the high-speed train," in 2013 European Control Conference (ECC), 2013, pp. 4568-4573.

[6] G. Mohd Riduwan, S. Mohd Helmi, I. Zuwairie, S. Muhammad Salihin, and M. T. Mohd Zaidi, "Single Input Fuzzy Logic Controller for Flexible Joint Manipulator," Int. J. Innov. Comput. Inf. Control, vol. 12, no. 1, pp. 181191, Feb. 2016.

[7] M. A. Ahmad, M. S. Saealal, R. M. T. R. Ismail, M. A. Zawawi, A. N. K. Nasir, and M. S. Ramli, "Single input fuzzy controller with command shaping schemes for double-pendulum-type overhead crane," AIP Conf. Proc., vol. 1337, no. 1, pp. 113-117, Jun. 2011.

[8] P. S. Londhe, M. Santhakumar, B. M. Patre, and L. M. Waghmare, "Task Space Control of an Autonomous Underwater Vehicle Manipulator System by Robust Single-Input Fuzzy Logic Control Scheme," IEEE J. Ocean. Eng., vol. 42, no. 1, pp. 13-28, Jan. 2017.

[9] K. Ishaque, Y. Saleem, S. S. Abdullah, M. Amjad, M. Rashid, and S. Kazi, "Single input fuzzy logic controller for magnetic levitation system," in Simulation and Applied Optimization 2011 Fourth International Conference on Modeling, 2011, pp. 1-6.

[10] B.-J. Choi, S.-W. Kwak, and B. K. Kim, "Design and stability analysis of single-input fuzzy logic controller," IEEE Trans. Syst. Man Cybern. Part B Cybern., vol. 30, no. 2, pp. 303-309, Apr. 2000.

[11] N. S. A. Shukor, M. A. Ahmad, and M. Z. M. Tumari, "Data-driven PID tuning based on safe experimentation dynamics for control of liquid slosh," in 2017 IEEE 8th Control and System Graduate Research Colloquium (ICSGRC), 2017, pp. 62-66. 


\section{BIOGRAPHIES OF AUTHORS}

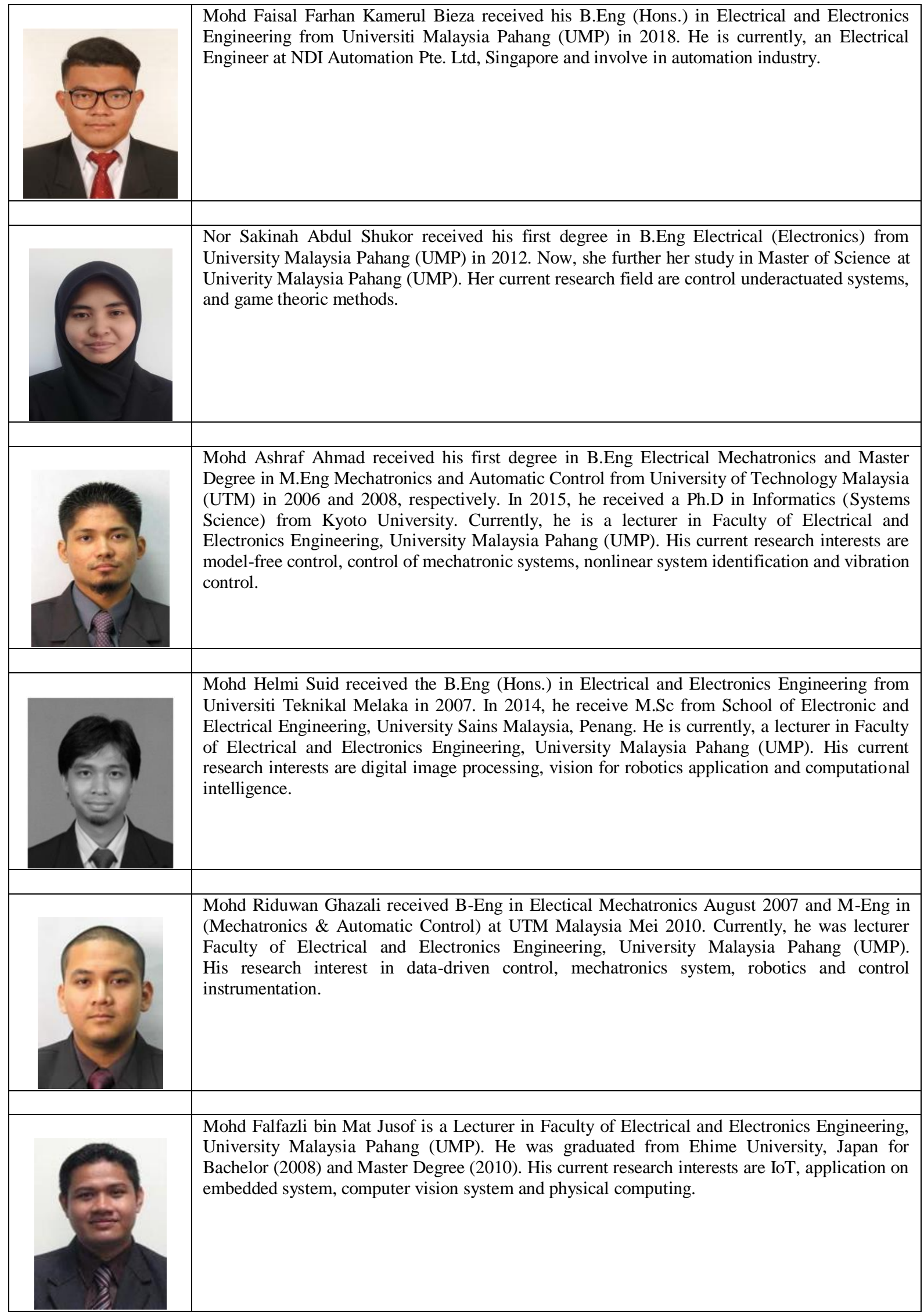

